\title{
Studies on Selective Adsorption Resins XXIX. Properties of Macroreticular Chelating Resins Containing Amidoxime Groups from Chloromethylstyrene-Divinylbenzene Copolymer Beads
}

\author{
Hiroaki Egawa, Takamasa Nonaka, and Kenji Tsukamoto \\ Department of Applied Chemistry, Faculty of Engineering, Kumamoto University, \\ Kumamoto-shi, Kumamoto 860, Japan
}

(Received November 22, 1990)

\begin{abstract}
Macroreticular chelating resins (RCSNHs) containing amidoxime groups were prepared from chloromethylstyrene-divinylbenzene copolymer beads (RCSs). The resins (RCCSNHs) having higher density of amidoxime groups were prepared from chloromethylated RCSs. The influence of specific surface area, pore volume, pore radius and density of amidoxime groups on adsorption ability for metal ions was investigated. Adsorption ability was greatly affected by pore structure of the resins. RCCSNHs showed higher adsorption ability than the corresponding RCSNHs prepared from the same RCSs. The density of amidoxime groups in the resins affected adsorption ability for metal ions.

KEY WORDS Chloromethylstyrene-Divinylbenzene Copolymer Bead / Amidoxime Group / Chelating Resin / Macroreticular / Adsorption of Metal Ion /
\end{abstract}

In recent years, chelating resins have been used for the selective removal and recovery of heavy metal ions from industrial waste solution containing much other cation. ${ }^{1}$ Previously, ${ }^{2}$ we prepared macroreticular chelating resins (RCSNHs) containing amidoxime groups from chloromethylstyrene(CMS)-divinylbenzene(DVB) copolymer beads (RCSs) and reported that RCSNHs were very effective for the recovery and separation of metal ions, the adsorption ability of RCSNHs for metal ions was greatly affected by pore structure of the resins and RCSNHs have high resistance against acid solution.

This article is concerned with the preparation of the resins (RCCSNHs) having high density of amidoxime groups from chloromethylated RCSs and the influence of specific surface area, pore volume, pore radius and the density of amidoxime groups in resins on adsorption ability for metal ions.

\section{EXPERIMENTAL}

Preparation of Macroreticular Chelating Resins Macroreticular chelating resins with various pore structures containing amidoxime groups were prepared by the route in Scheme 1.

Preparation of RCSNH: RCS was synthesized by suspension copolymerization of the chloromethylstyrene and divinylbenzene in the presence of cyclohexane as a diluent. The polymerization conversion was about $97 \%$. Divinylbenzene (DVB) was used as technical grade (containing 57\% DVB). Nitrile groups were introduced to RCS, and the product (RCSN) was treated with hydroxylamine in methanol at $80^{\circ} \mathrm{C}$ for $10 \mathrm{~h}$ by the same method described previously. ${ }^{2}$

Preparation of RCCSNH: RCS $(5 \mathrm{~g})$ was chloromethylated with chloromethylether (31.4 $\mathrm{ml}$ ) at $0-5^{\circ} \mathrm{C}$ for $3 \mathrm{~h}$ in the presence of tetrachloroethane $(3.1 \mathrm{ml})$ as a swelling agent 


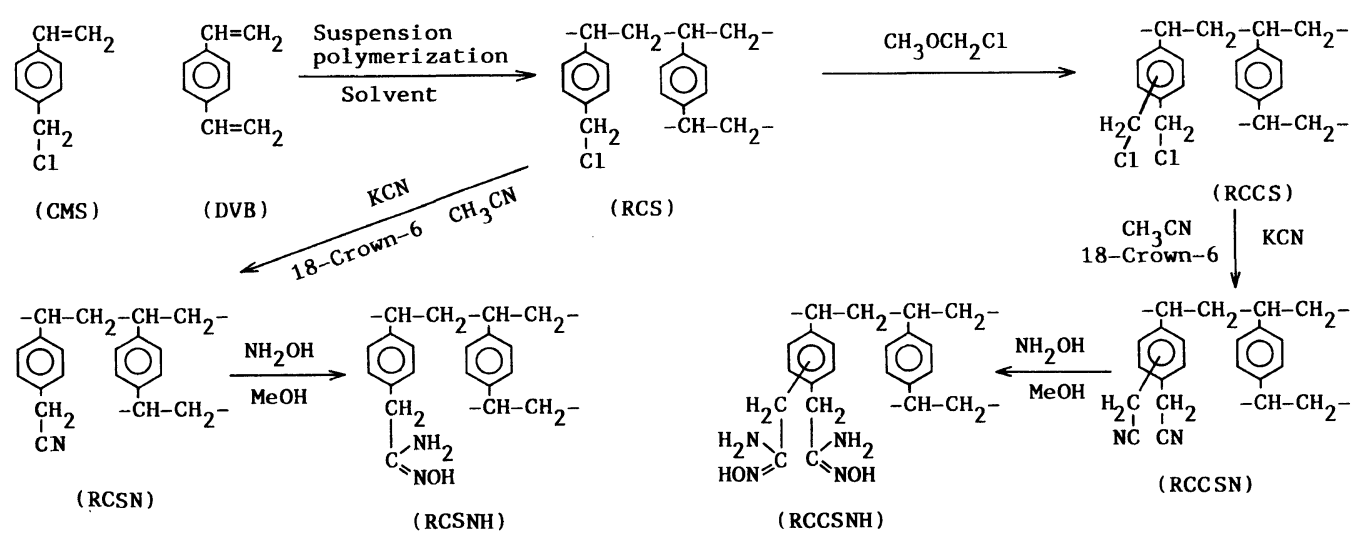

Scheme 1. Preparation of RCSNH and RCCSNH.

and aluminium chloride $(6.7 \mathrm{~g})$ as catalyst. The chlorine content of the resins was determined by the flask combustion method. ${ }^{3}$ Nitrile groups were introduced to the RCCS ( $1 \mathrm{~g}$ ) by reaction with $\mathrm{KCN}(3.2 \mathrm{~g})$ in acetonitrile $(50 \mathrm{ml})$, using 18-crown-6 $(3.2 \mathrm{~g})$ as a phase transfer catalyst, for $24 \mathrm{~h}$ under reflux. The product (RCCSN) was treated with hydroxylamine in methanol at $80^{\circ} \mathrm{C}$ for $10 \mathrm{~h}$ to obtain RCCSNH.

\section{Measurement of Pore Structures}

Specific surface areas of resins obtained were measured on Yuasa surface area apparatus (BET method). Pore volume and radius of the resins were determined on Carlo-Erba mercury porosimeter (Model 220).

\section{Measurement of Adsorption Capacity for Metal Ions}

The resin $(0.125 \mathrm{~g})$ and $50 \mathrm{~cm}^{3}$ of a 0.01 mol dm ${ }^{-3}$ metal ion solution were shaken in a glass-stoppered erlenmayer flask at $30^{\circ} \mathrm{C}$ for $24 \mathrm{~h}$. The amounts of metal ions absorbed on the resin were determined by chelatometric titration of metal ions in the supernatant. The metal ion solution was prepared from nitrates of reagent grade.

\section{Adsorption of Uranium from Uranium-Spiked} Seawater

The resin $(0.1 \mathrm{~g})$ and $25 \mathrm{~cm}^{3}$ of U-spiked seawater $\left(494 \mu \mathrm{g}\right.$ of $\mathrm{UO}_{2}\left(\mathrm{NO}_{3}\right)_{2} \cdot 6 \mathrm{H}_{2} \mathrm{O}$ were added to it) were shaken at $30^{\circ} \mathrm{C}$. The percentage of uranium adsorbed on the resin was calculated from spectrophotometric determination of uranium in the supernatant with Arsenazo-III.

\section{RESULTS AND DISCUSSION}

\section{Preparation of Macroreticular Chelating Resins}

In order to obtain chelating resins with various macroreticular structures, RCSs were prepared using various contents of cyclohexane $(50-160 \mathrm{vol} \%$ to monomer) and constant DVB-total monomers $(1: 10 \mathrm{~mol}$ ratio $)$ in suspension polymerization. RCSNHs and RCCSNHs were prepared from these RCSs and chloromethylated RCSs. Table I shows the properties of the resins. The $\mathrm{Cl}$ content of RCS was smaller than that calculated $(19.9 \%)$. This decrease in $\mathrm{Cl}$ content is attributed to the hydrolysis of the chloromethyl groups during suspension polymerization. ${ }^{2}$ The $\mathrm{Cl}$ content of RCS increased by chloromethylation.

Table I shows also that RCSNHs have $2.5-2.9 \mathrm{meq} \mathrm{g}^{-1}$ of anion exchange capacity and RCCSNHs have $3.4-3.7 \mathrm{meq}^{-1}$. This indicates that the density of amidoxime groups 
Table I. Properties of RCS, RCSNH, RCCS, and RCCSNH

\begin{tabular}{|c|c|c|c|c|c|c|}
\hline \multirow{3}{*}{$\begin{array}{c}\text { Cyclohexane } \\
\text { vol } \%\end{array}$} & \multirow{3}{*}{$\begin{array}{c}\mathrm{RCS} \\
\mathrm{Cl} \\
\mathrm{wt} \%\end{array}$} & \multicolumn{2}{|c|}{ RCSNH } & \multirow{3}{*}{$\begin{array}{c}\text { RCCS } \\
\mathrm{Cl} \\
\%\end{array}$} & \multicolumn{2}{|c|}{ RCCSNH } \\
\hline & & $\mathrm{Ca}$ & $\mathrm{Cc}$ & & $\mathrm{Ca}$ & $\mathrm{Cc}$ \\
\hline & & meq $g^{-1}$ & $\operatorname{meq~g~}^{-1}$ & & meq $g^{-1}$ & meq $g^{-1}$ \\
\hline 50 & 15 & 2.8 & 0.1 & 28 & 3.6 & 0.4 \\
\hline 75 & 15 & 2.9 & 0.1 & 27 & 3.7 & 0.5 \\
\hline 100 & 14 & 2.9 & 0.1 & 26 & 3.5 & 0.4 \\
\hline 120 & 14 & 2.5 & 0.2 & 28 & 3.5 & 0.4 \\
\hline 140 & 15 & 2.8 & 0.1 & 26 & 3.4 & 0.4 \\
\hline 160 & 14 & 2.5 & 0.2 & 26 & 3.4 & 0.4 \\
\hline
\end{tabular}

$\mathrm{Cl}, \mathrm{Cl}$ content.

$\mathrm{Ca}$, Anion exchange capacity.

$\mathrm{Cc}$, Cation exchange capacity.

Table II. Specific surface area, pore volume, and pore radius

\begin{tabular}{|c|c|c|c|c|c|c|}
\hline \multirow[b]{2}{*}{ Cyclohexane } & \multicolumn{3}{|c|}{ RCSNH } & \multicolumn{3}{|c|}{ RCCSNH } \\
\hline & Specific & Pore & Average & Specific & Pore & Average \\
\hline \multirow{2}{*}{ vol $\%$} & surface area & volume & pore radius & surface area & volume & pore radius \\
\hline & $\mathrm{m}^{2} \mathrm{~g}^{-1}$ & $\mathrm{~cm}^{3} \mathrm{~g}^{-1}$ & $\AA$ & $\mathrm{m}^{2} \mathrm{~g}^{-1}$ & $\mathrm{~cm}^{3} \mathrm{~g}^{-1}$ & $\AA$ \\
\hline 50 & 0.1 & - & - & 0.2 & - & - \\
\hline 75 & 7.5 & - & - & 6.9 & - & - \\
\hline 100 & 35.5 & 0.239 & 123 & 41.2 & 0.203 & 138 \\
\hline 120 & 56.3 & 0.935 & 271 & 58.0 & 0.857 & 319 \\
\hline 140 & 35.3 & 1.386 & 778 & 39.0 & 1.278 & 854 \\
\hline 160 & 13.7 & 1.735 & 3354 & 15.2 & 1.636 & 3561 \\
\hline
\end{tabular}

in RCCSNH is higher than that in RCSNH. In the previous article, we reported that almost $100 \%$ of the chloromethyl groups were converted to nitrile groups, followed by about $70 \%$ conversion to the amidoxime groups. The specific surface area, pore volume, and average pore radius of the resins are illustrated in Table II. In the use of 50 and $75 \mathrm{vol} \%$ of cyclohexane, the resins obtained were gel type resins with no or small specific surface area. On the other hand, resins with macroreticular structure could be prepared by using $100-160 \mathrm{vol} \%$ of cyclohexane. Pore volume and average pore radius of the resins increased with increasing volume percent of cyclohexane. The specific surface area of the resins increased with increasing volume percent of cyclohexane up to $120 \mathrm{vol} \%$. In the use of above $120 \mathrm{vol} \%$, specific surface area decreased with increasing cyclohexane content. Large differences of specific surface area, pore volume and average pore radius were not observed between RCSNHs and corresponding RCCSNHs prepared by using the same RCS.

\section{Adsorption Ability for Metal Ions}

The adsorption capacity of resins (RCSNHs and $\mathrm{RCCSNHs}$ ) for $\mathrm{Cu}^{2+}, \mathrm{UO}_{2}{ }^{2+}$, and $\mathrm{Ag}^{+}$ was measured (Figure 1). RCSNHs prepared by using 50 and $75 \mathrm{vol} \%$ of cyclohexane could adsorb very little $\mathrm{Cu}^{2+}$ and no $\mathrm{UO}_{2}{ }^{2+}$, although they had almost the same anion exchange 

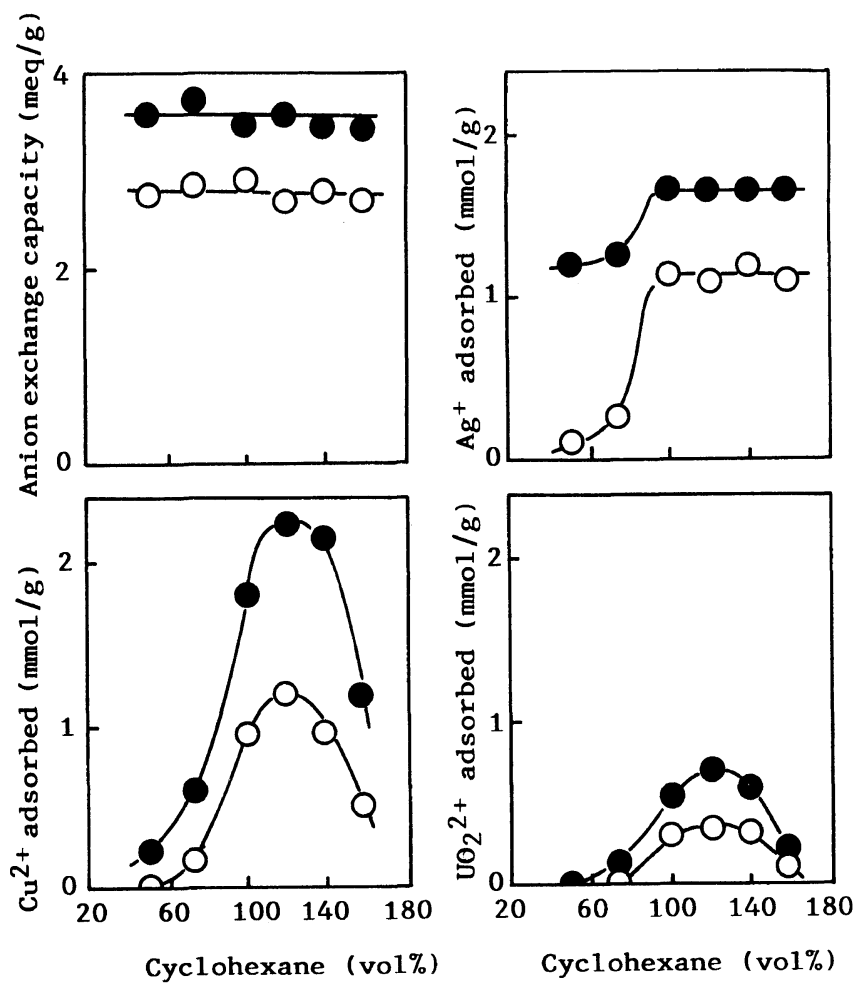

Figure 1. Effects of pore structure and density of amidoxime groups on the adsorption of metal ions: O, RCSNH; ○, RCCSNH.

capacity as RCSNHs prepared by using the above $100 \mathrm{vol} \%$ of cyclohexane. $\mathrm{UO}_{2}{ }^{2+}$ and $\mathrm{Cu}^{2+}$ were adsorbed most on the RCSNH and RCCSNH prepared by using $120 \mathrm{vol} \%$ of cyclohexane. Adsorption of $\mathrm{Ag}^{+}$on the RCSNH prepared by using below $75 \mathrm{vol} \%$ of cyclohexane decreased remarkably. However $\mathrm{Ag}^{+}$was slightly adsorbed on the resins. This indicates that metal ions such as $\mathrm{Cu}^{2+}$ or $\mathrm{UO}_{2}{ }^{2+}$ which have high radius of its hydrated ion are very difficult to diffuse to the inner part of resins which have no macroreticular structure. This, it was found that the adsorption of $\mathrm{Cu}^{2+}$ and $\mathrm{UO}_{2}{ }^{2+}$ was greatly affected by the pore structure, especially by the specific surface area of the resins. All RCCSNHs adsorbed more metal ions than the corresponding RCSNHs. A large difference in adsorption capacity for $\mathrm{Cu}^{2+}$ and $\mathrm{UO}_{2}{ }^{2+}$ was observed between RCSNHs and RCCSNHs prepared by using $120 \mathrm{vol} \%$ of cyclohexane. The large increase in adsorption capacity for $\mathrm{Ag}^{+}$of the RCCSNHs prepared by using 50 and $75 \mathrm{vol} \%$ of cyclohexane was also observed. This is due to increase in the density of amidoxime groups and hydrophilicity of the resin which makes the diffusion and adsorption of each metal ion easier. These results are supported by the observation of the distribution of metal ions adsorbed on the resins, measured by an electron-probe microanalyzer (Figure 2).

In the case of $\mathrm{RCSNH}$ prepared by using $50 \mathrm{vol} \%$ of cyclohexane, $\mathrm{UO}_{2}{ }^{2+}$ and $\mathrm{Cu}^{2+}$ were hardly adsorbed on the resin and $\mathrm{Ag}^{+}$ was adsorbed only on the surface parts of resin particles. But in the case of RCCSNH prepared from the same $\mathrm{RCS}, \mathrm{UO}_{2}{ }^{2+}$ and $\mathrm{Cu}^{2+}$ were 

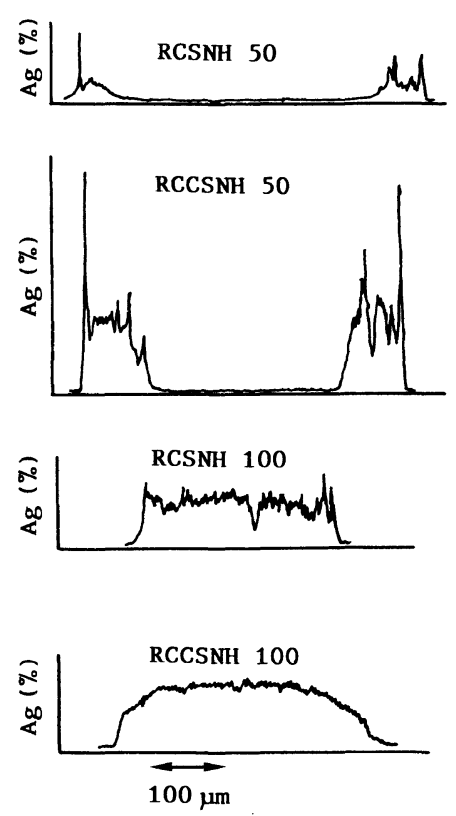
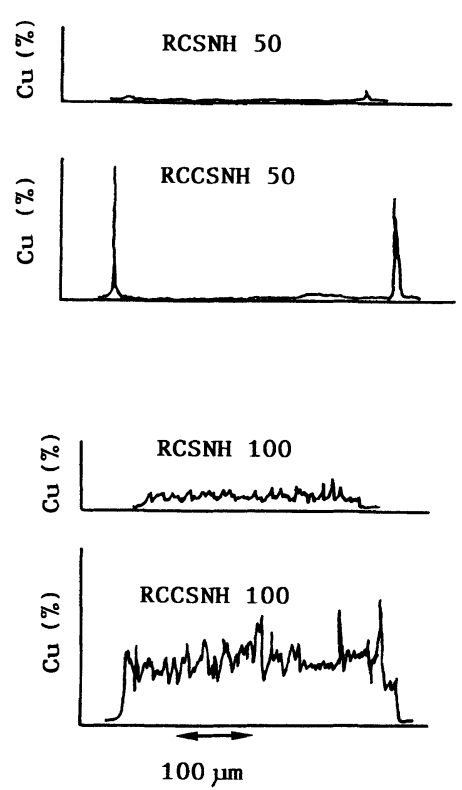
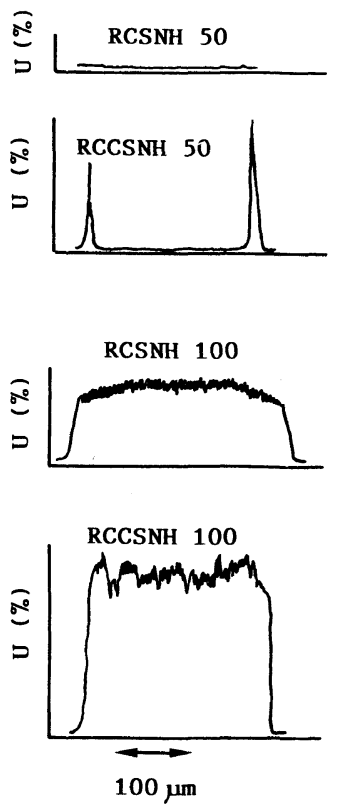

Figure 2. Distribution of metal ions adsorbed on the resins.

adsorbed slightly on the surface of the resin and $\mathrm{Ag}^{+}$was adsorbed from the surface to inner part of the resin. It was found that both RCSNH and RCCSNH prepared by using $100 \mathrm{vol} \%$ of cyclohexane adsorbed each metal ion throughout the resin particles.

The adsorption ability of resins for uranium in uranium-spiked seawater was investigated (Figure 3). The RCSNH prepared by using $50 \mathrm{vol} \%$ of cyclohexane showed little adsorption for uranium even after $75 \mathrm{~h}$. But RCCSNH prepared from the same RCS adsorbed 25\% of uranium in the solution. RCCSNHs showed higher adsorption rates and adsorption percent for uranium than the corresponding RCSNHs.

\section{Effects of pH on the Adsorption of Metal Ions}

The adsorption capacity of RCSNH and RCCSNH prepared by using $10 \mathrm{~mol} \%$ of DVB and $120 \mathrm{vol} \%$ of cyclohexane for various metal ions in aqueous solution with different $\mathrm{pH}$ was investigated (Figure 4). The $\mathrm{pH}$ in the abscissa are equilibrium values after adsorption. Both RCSNH and RCCSNH have high adsorption
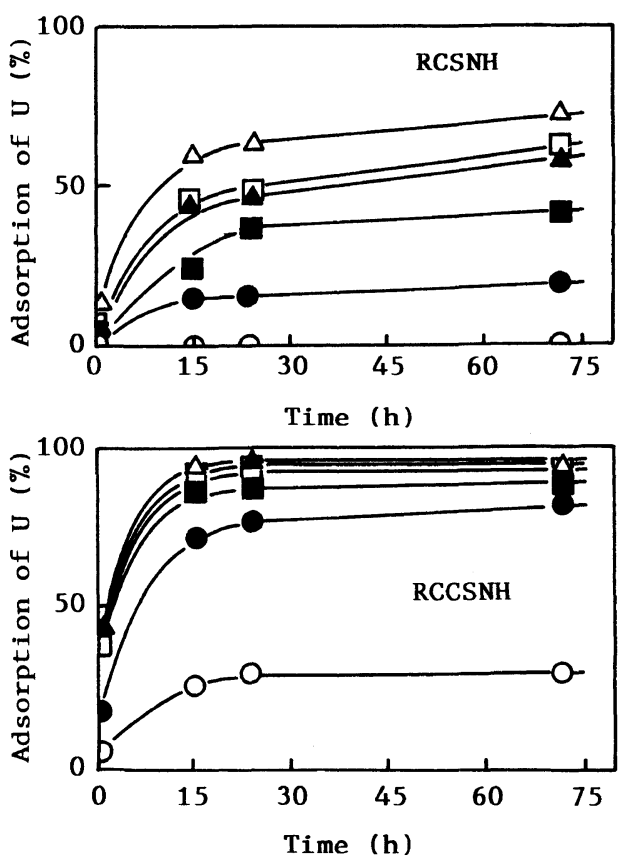

Figure 3. Effects of pore structure on the adsorption of uranium from U-spiked seawater: Cyclohexane/vol\%; $\bigcirc$, $50 ; \bigcirc, 75 ; \square, 100 ; \triangle, 120 ; \mathbf{\Delta}, 140 ; \square, 160$. 

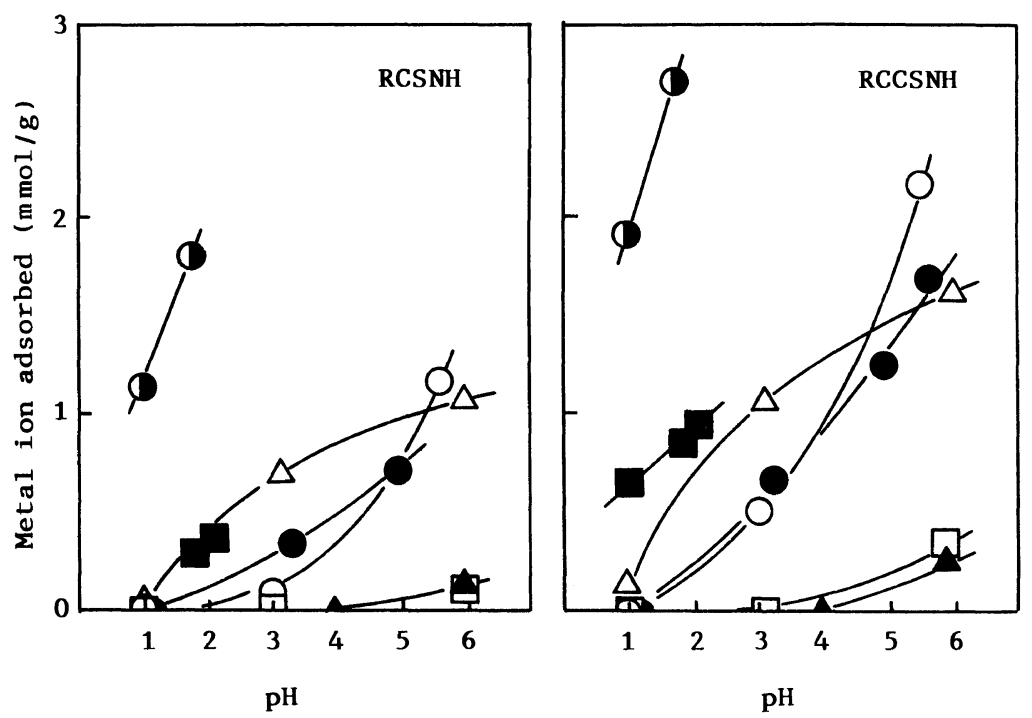

Figure 4. Adsorptions of various metal ions: $\mathrm{C}, \mathrm{Hg}^{2+} ; \triangle, \mathrm{Ag}^{+}$ $\mathrm{Zn}^{2+}$; $\mathrm{UO}_{2}{ }^{2+}$.
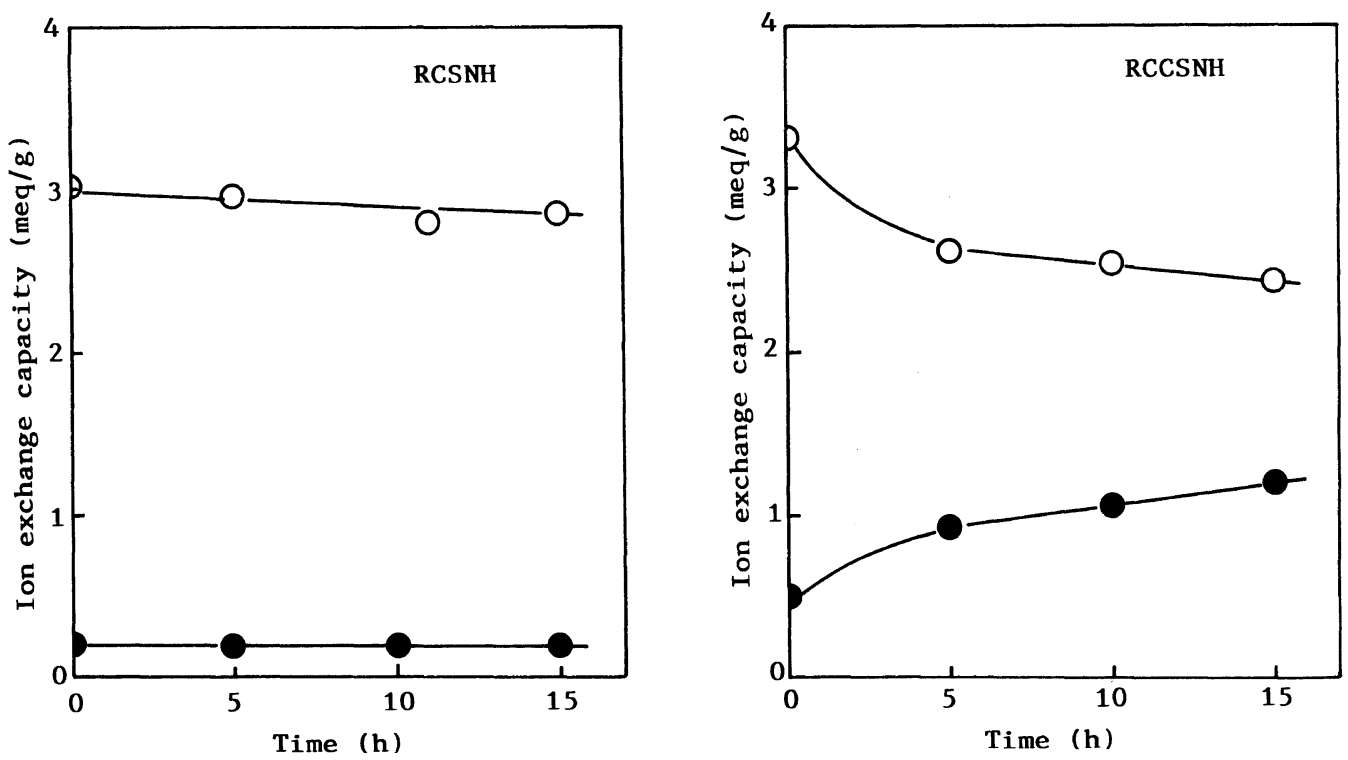

Figure 5. Effects of acid treatment on ion exchange capacity: $\bigcirc$, anion exchange capacity; exchange capacity.

capacity for $\mathrm{Hg}^{2+}$ in solution with low $\mathrm{pH}$ (1-2). RCSNH showed adsorption ability for $\mathrm{Ag}^{+}$and $\mathrm{Fe}^{3+}$ in the solution with $\mathrm{pH} 2$, whereas RCCSNH was able to adsorb them at $\mathrm{pH} 1 . \mathrm{Cu}^{2+}$ and $\mathrm{UO}_{2}{ }^{2+}$ could be adsorbed from the solution at $\mathrm{pH}$ above $3 . \mathrm{Pb}^{2+}$ and $\mathrm{Zn}^{2+}$ could hardly be adsorbed on $\mathrm{RCSNH}$ even at pH 6. RCCSNH showed higher adsorption capacity for various metal ions than RCSNH. 


\section{Resistance against Acid Solution}

We reported that RCSNHs have high resistance against acid solution. The changes of anion exchange capacity of resins after acid treatment with $1 \mathrm{moldm}^{-3} \mathrm{HCl}$ at $60^{\circ} \mathrm{C}$ are shown in Figure 5. The changes in ion exchange capacity of RCSNH could hardly be observed. But, in the case of RCCSNH, the anion exchange capacity decreased and the cation exchange capacity increased with increase in the time of acid treatment. This suggests that the amidoxime groups decrease and acidic groups such as carboxyl groups etc. are formed by acid treatment. These results indicate that RCCSNHs are slightly inferior to RCSNHs in resistance against acid solution. At the present, it is not clear that why different resistance against acid solution was observed. This needs much future study.

\section{CONCLUSIONS}

The adsorption ability (capacity and rate) of RCSNHs and RCCSNHs for metal ions such as $\mathrm{Cu}^{2+}$ and $\mathrm{UO}_{2}{ }^{2+}$ was not only affected by the density amidoxime groups but also by the pore structure of the resins. Especially, the order of adsorption capacity for $\mathrm{Cu}^{2+}$ and $\mathrm{UO}_{2}{ }^{2+}$ of the macroreticular RCSNHs and RCCSNHs corresponded to that of specific surface area of the resins. RCCSNHs having higher density of amidoxime groups showed higher adsorption ability for metal ions than the corresponding RCSNHs prepared from the same RCS. It was found that both RCSNHs and RCCSNHs having high porous structure uniformly adsorbed each metal ion throughout the resin particles.

Acknowledgments. This work was supported by a Grant-in-Aid for Scientific Research on Priority Area of "Dynamic Interactions and Electronic Processes of Macromolecular Complexes" (No. 63612001) from the Ministry of Education, Science, and Culture of Japan.

\section{REFERENCES}

1. H. Egawa, Kagaku to Kogyo, 63, 392 (1989).

2. H. Egawa, T. Nonaka, and K. Tsukamoto, Polym. J., 22, 120 (1990).

3. Yuki Biryo Bunseki Kenkyu Kondankai, Ed., "Yuki Biryo Teiryo Bunseki," Nankodo, Tokyo, 1969, p 383. 